\title{
Development of MEMS-IMU/GPS Integrated Navigation System
}

\author{
Jeong Won Kim¹, Chang Woo Nam, Jae-Cheul Lee', Sung Jin Yoon², Jaewook Rhim² \\ ${ }^{1}$ Hanwha Corporation R\&D Center, Daejeon 305-156, Korea \\ ${ }^{2}$ Agency for Defense Development, Daejeon 305-600, Korea
}

\begin{abstract}
In the guided missile and unmanned vehicle system, the navigation system is one of the most important components. Recently, low-cost effective smart projectiles and guided bomb are being developed using MEMS based navigation system which has high-G, low-cost and small size. In this paper, locally developed MEMS based GPS/INS integrated navigation system will be introduced in comparison with the state of the art of MEMS based navigation system. And technical design and development method is described to satisfy the required performance of GPS receiver, MEMS inertial sensor assembly, navigation computer and software.
\end{abstract}

Keywords: MEMS, GPS/INS integrated navigation system

\section{INTRODUCTION}

A navigation system provides position information to guided weapon systems, unmanned systems, aircraft, ships, and vehicles; and it is an important element that determines the system performance. Navigation systems are divided into an inertial navigation system and a satellite navigation system. The inertial navigation system estimates position, velocity, and attitude through the integration of the angular velocity and acceleration measured by a gyroscope and an accelerometer, which are inertial sensors. The satellite navigation system, which is represented by the Global Positioning System (GPS) of the United States, estimates position by measuring the arrival times of the radio signals broadcast from satellites and by using the principle of triangulation.

Inertial navigation systems are classified depending on the type and performance of inertial sensors. They are classified into the Gimbal type, where the axis of the inertial sensor is always consistent with the navigation frame, and the Strapdown type, where the axis of the inertial sensor is

Received Mar 31, 2014 Revised May 08, 2014 Accepted May 09, 2014 tCorresponding Author

E-mail: jwkim76@hanwha.com

Tel: +82-42-828-0533 Fax: +82-42-828-0420 consistent with the body frame; and can also be classified depending on the principle of the inertial sensor, such as mechanical gyroscope, optical gyroscope, and laser gyroscope. As an inertial navigation system calculates position by the principle of integration, the error of the inertial sensor accumulates, and the error diverges with time. To obtain accurate navigation information, a highperformance inertial sensor should be used. However, it has the disadvantages of increased price, power consumption, and volume, in proportion to the performance.

Recently, guided weapons that improve accuracy while pursuing cost efficiency by adding guidance/navigation/ control functions to free-fall bombs and gun-launched projectiles have been developed. The representative weapon systems include smart projectiles such as Joint Direct Attack Munitions (JDAM), Extended Range Guided Munitions (ERGM), and Excalibur.

The navigation system for these guided weapon systems should satisfy small size, low power, low cost, and highshock survivability. However, existing mechanical and optical sensors cannot satisfy these conditions. With the recent development of Micro ElectroMechanical System (MEMS) technology, inertial sensors that can satisfy these conditions have been developed (Dowdle \& Flueckiger 1996, Barbour 2001, Warnasch \& Killen 2002, Brown 2003, Habibi et al. 2008). An MEMS inertial sensor has a number of advantages, but has lower performance than mechanical 
and optical sensors. Thus, it is difficult to independently construct a navigation system, and it is generally developed in the form of a GPS/INS integrated navigation system, through combination with a GPS receiver (Schmidt 1999). The representative MEMS-based GPS/INS integrated navigation systems include the IGS-200 of Honeywell International, Inc. from the United States and the SiNav of AIS, Inc. from the United Kingdom. Their performances have already been verified by applying them to weapon systems. An MEMS-based navigation system can be used for various applications in addition to guided bombs and smart projectiles. In Korea, the development of a number of guided weapons using MEMS-IMU or navigation system has also been performed, and it is expected to be used in more diverse fields in the future.

This paper introduces the status of the development of an MEMS-based GPS/INS integrated navigation system in Korea. The design method and applied technique of the MEMS-IMU/GPS integrated navigation system are described; and the developed result and its performance are introduced. For the content of this paper, the status of the development of navigation systems in foreign countries is introduced in Section 2; and the design content, development result, and performance of the MEMS-IMU/ GPS integrated navigation system are described in Sections 3 and 4 . Lastly, future directions are suggested.

\section{STATUS OF MEMS-IMU/GPS INTEGRATED NAVIGATION SYSTEMS IN FOREIGN COUNTRIES}

The representative MEMS-IMU/GPS integrated navigation systems include the IGS-200 of Honeywell International, Inc. from the United States and the SiNav of AIS, Inc. from the United Kingdom. In addition, there are a number of integrated navigation systems developed based on commercial MEMS sensors. However, most of them have very low performance, and their reliability and environmental resistance are insufficient for weapon systems. In Table 1, the performances of the representative MEMS-IMU/GPS integrated navigation systems were compared. As summarized in Table 1, the MEMS-IMU/GPS integrated navigation systems have a small size; the biases of the gyroscope and the accelerometer are 20 50 deg/hr and 2.5 5 mg, respectively; a position accuracy performance of within $10 \mathrm{~m}$ can be provided through integrated navigation with GPS; and the J/S values are higher than that of a general GPS receiver. Especially, the IGS-200 has high electromagnetic wave disturbance resisting performance due to the application of a nulling technique.

\section{DESIGN AND DEVELOPMENT OF AN MEMS-IMU/GPS INTEGRATED NAVIGATION SYSTEM}

An MEMS-IMU/GPS integrated navigation system was designed so that reliable and accurate navigation information could be provided, by sufficiently reflecting the advantages of an MEMS inertial sensor and by complementing the disadvantages of an MEMS sensor via integrated navigation. Table 2 summarizes the design directions.

As shown in Fig. 1, the MEMS-IMU/GPS integrated navigation system consists of inertial sensor assembly (ISA), signal processing board, navigation computer, GPS receiver, EMI filter board, housing, and vibration isolator. For size minimization, all the electronic components were designed to have a diameter of less than $60 \mathrm{~mm}$ by increasing the

Table 1. Performances of the MEMS-IMU/GPS integrated navigation systems in foreign countries.

\begin{tabular}{|c|c|c|}
\hline Item & IGS-200 (by Honeywell, US) & SiNAV (by AIS, UK) \\
\hline \multirow[b]{2}{*}{ Shape } & $\stackrel{83 \mathrm{~mm}}{\longrightarrow}$ & $80 \mathrm{~mm}$ \\
\hline & & \\
\hline Bias repeatability [deg/hr] & 20 & 50 \\
\hline Noise $[\mathrm{deg} / \sqrt{\mathrm{hr}}]$ & 0.125 & 0.5 \\
\hline Bias repeatability [mg] & 5 & 2.5 \\
\hline Noise $[\mathrm{m} / \mathrm{s} / \sqrt{\mathrm{hr}}]$ & 0.09 & 0.5 \\
\hline Frequency & GPS L1/L2 (P) & GPS L1 (C/A) \\
\hline Position accuracy [m] & 5 (CEP) & $10(\mathrm{CEP})$ \\
\hline $\mathrm{J} / \mathrm{S}[\mathrm{dB}]$ & $>95$ & $40 \sim 45$ \\
\hline Size $[\mathrm{mm}] /$ Weight $[\mathrm{g}] /$ Power $[\mathrm{W}]$ & $71(\mathrm{D}) \times 67(\mathrm{H}) / 567 /<10$ & 80 (D) $\times 72(\mathrm{H}) /<400 /<8$ \\
\hline Interface & SDLC / RS-422 & SDLC / RS- 422 \\
\hline
\end{tabular}


Table 2. Design directions of the MEMS-IMU/GPS integrated navigation system.

\begin{tabular}{|c|c|}
\hline Item & Design direction \\
\hline \multirow{2}{*}{$\begin{array}{l}\text { Size minimization/ } \\
\text { Weight lightening } \\
\text { Accuracy }\end{array}$} & $\begin{array}{l}\text { - Device minimization through ASIC of the major } \\
\text { modules }\end{array}$ \\
\hline & $\begin{array}{l}\text { - High-integration/high-density HW design } \\
\text { - High-performance MEMS inertial sensor } \\
\text { development } \\
\text { - Accuracy improvement through deep coupling } \\
\text { with GPS }\end{array}$ \\
\hline Efficiency & $\begin{array}{l}\text { - HW usage efficiency improvement through } \\
\text { the application of single CPU architecture and } \\
\text { programmable devices }\end{array}$ \\
\hline $\begin{array}{l}\text { Environmental } \\
\text { resistance }\end{array}$ & $\begin{array}{l}\text { - Design for satisfying the resistance to harsh } \\
\text { environments among the military standards }\end{array}$ \\
\hline $\begin{array}{l}\text { High-impact } \\
\text { survivability }\end{array}$ & $\begin{array}{l}\text { - Application of the mechanical structure and } \\
\text { parts for high-impact resistance }\end{array}$ \\
\hline
\end{tabular}

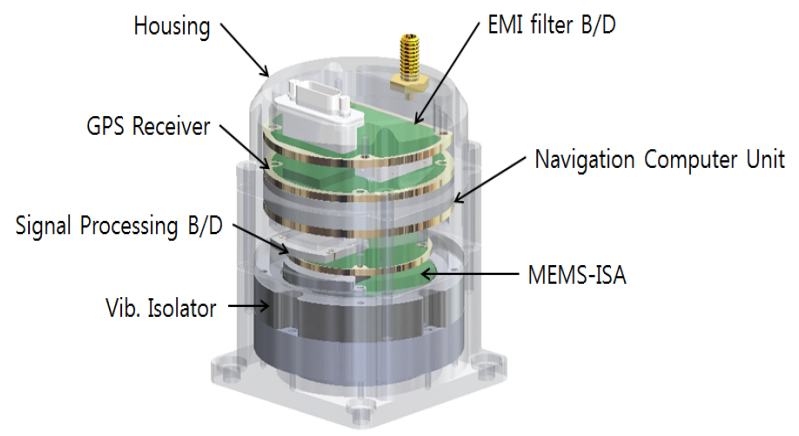

Fig. 1. Structure of the MEMS-IMU/GPS integrated navigation system.

integration density using multi-layer PCB manufactured by a build-up process, and the major modules were manufactured by ASIC. The efficiency of HW resource usage was increased by the development of an MEMS inertial sensor that has high-grade performance among the grades of MEMS, by the accurate navigation information providing function through deep coupling with GPS, and by the application of single CPU architecture and programmable devices. Also, it was designed to satisfy military environment standards so that the environmental resistance appropriate for weapon systems could be obtained; and the structure was designed so that it could tolerate harsh environments such as gun-launching high impact.

The MEMS ISA consists of an MEMS sensor structure and electronic circuits that control the structure and perform signal processing. The gyroscope of the MEMS is a vibration ring type, and the accelerometer is an electrostatic capacity type. The size of the structure is very small ( $\mathrm{OxO} \mathrm{mm}$ ), and the performance was secured by vacuum packaging. The electronic circuits of the gyroscope consist of preamplifier, analog to digital converter (ADC), digital to analog converter (DAC), field-programmable gate array (FPGA), and frequency tuning circuit. The preamplifier is a circuit that

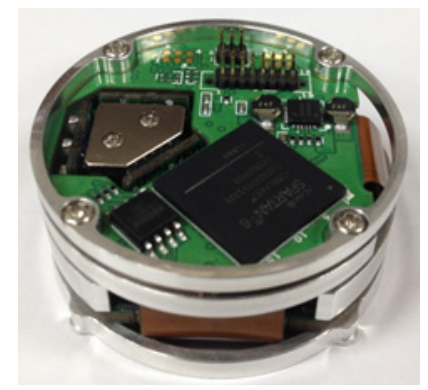

Fig. 2. Shape of the MEMS ISA.

converts the change in electrostatic capacity, which occurs due to the displacement of a sensing structure, into a voltage. To sense minute changes in electrostatic capacity, a lownoise design was applied. The output of the preamplifier is converted to a digital signal via the differential amplifier and ADC. Then, it is entered into FPGA, and is used as an input signal for the four control loops within FPGA, respectively. Among the four control circuits, the magnetic resonance control circuit and automatic gain control circuit control the vibration of the driving axis, and the rebalance control circuit and quadrature control circuit control the vibration of the sensing axis. If Coriolis force is applied to the sensing axis due to angular velocity, the rebalance control circuit controls so that the sensing axis can achieve force balance, by applying a force whose magnitude is identical to that of the Coriolis force in the anti-phase. The quadrature control circuit was designed so that it could control the quadrature vibration that occurs at the sensing axis due to frequency offset and external vibration. The electronic circuit of the accelerometer was designed as an analog voltage control structure that can be used in a high acceleration range, through the analysis of existing methods. The electrostatic capacity sensing circuit used a preamplifier with an amplitude modulation charge amplification method, and was designed as a structure that performs feedback control without distinguishing a sensing electrode and a driving electrode. The control circuits excluding the amplifier, ADC, and DAC were designed in a digital method, and the size of the ISA was minimized through implementation using FPGA. Fig. 2 shows the shape of the developed MEMS-ISA.

The signal processing board performs the real-time correction of the fixed error and temperature-dependent error included in the sensor signal of the MEMS ISA, and outputs the inertial measurements for navigation operation through coning/sculling compensation. To perform many operations of real numbers at a high speed, the signal processing board was designed based on a digital signal processor (DSP) series processor. For the external 


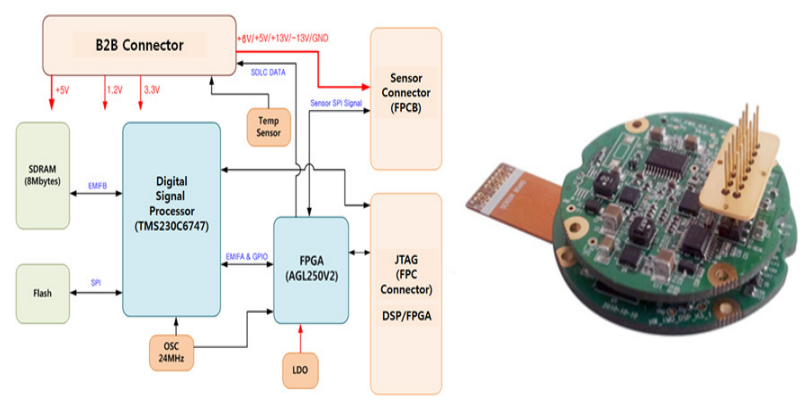

Fig. 3. Structure and shape of the MEMS signal processing board.

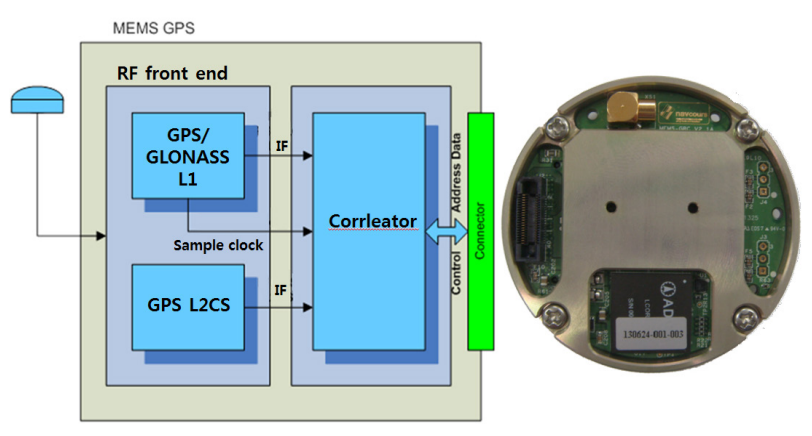

Fig. 4. Structure and shape of the GPS receiver.

communication interface, an SDLC method was applied, and flexibility was obtained through implementation based on FPGA. Fig. 3 shows the structure and shape of the signal processing board. For size minimization, the diameter of the signal processing board was made to be less than $40 \mathrm{~mm}$.

The GPS receiver was designed to be able to process GPS L1/L2 and GLONASS L1 signals so that it could operate based on multi-band frequencies. For size minimization, the RF part and the correlator (i.e., digital signal processing part) were developed as 1 chip, respectively, through ASIC. OO-channel satellite signals can be received. The correlator has an extended range correlator (ERC) structure, which has wider tracking range than existing correlators. Also, for high-impact resistance, a clock that is robust to high impact was applied, and the RF part was protected with a can that has a disturbance shielding and mechanical reinforcement structure, as shown in Fig. 4. After the correlator of the GPS receiver, signal processing is performed by SW, and is operated in the navigation computer.

For an existing GPS/INS integrated navigation system, CPU for GPS SW and CPU for integrated navigation SW exist separately. The MEMS-IMU/GPS integrated navigation system was designed as a single CPU based architecture structure, and thus, one CPU operates GPS SW and integrated navigation SW in real time. Single CPU architecture has advantages in terms of size, power consumption, and cost; and is known to be an optimal $\mathrm{H} / \mathrm{W}$ structure for performing deeply coupled integrated navigation. For deeply coupled integrated navigation, the duplicate signal generator within the GPS receiver, a numerical controlled oscillator (NCO), should be controlled by inertial navigation and the output of the filter at a high refresh rate $(1000 \mathrm{~Hz})$, and the output of the correlator should be processed by a pre-processing filter or an integrated filter. If each CPU is used, data need to be exchanged via dual port RAM or serial/parallel communication method, and thus, the performance could deteriorate due to the implementation loss such as transmission delay. To operate GPS SW and integrated navigation SW in single CPU, the navigation computer was designed using high-performance CPU. The CPU is a PowerPC series, and has a small size and a high performance as it was manufactured by a $90 \mathrm{~nm}$ process. It provides various functions such as $400 \mathrm{MHz}$ DDR Memory Controller, USB Dual-Role Controller, Dual Enhanced Three-Speed Ethernet Controllers, Integrated Programmable Interrupt Controller, Power Management Controller, and Serial Peripheral Interface. In addition, it belongs to a product group that is included in the product longevity program of the manufacturer, and thus, the risk of being discontinued is low. The major module other than the CPU part is the external communication interface. All the functions were designed so that easy modification of the functions could be facilitated, through implementation using FPGA. The navigation computer board has a size of
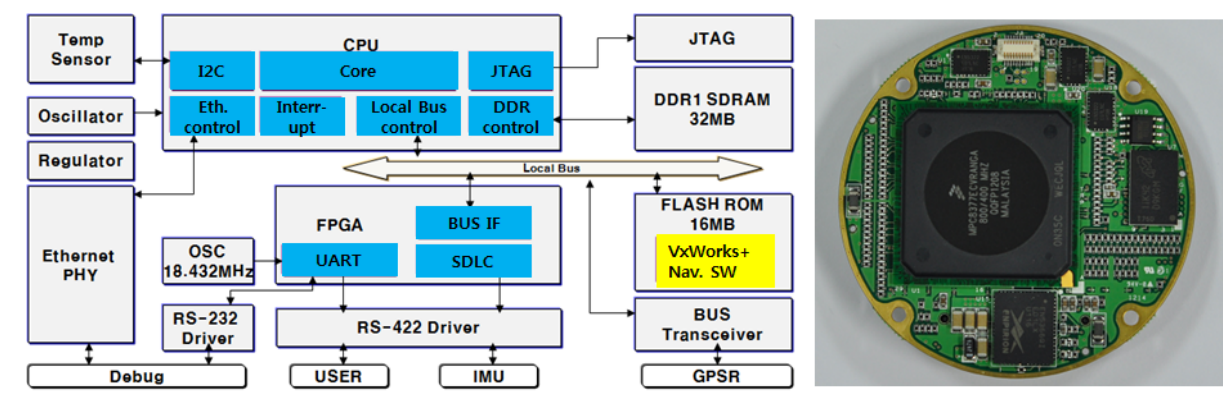

Fig. 5. Structure and shape of the navigation computer. 


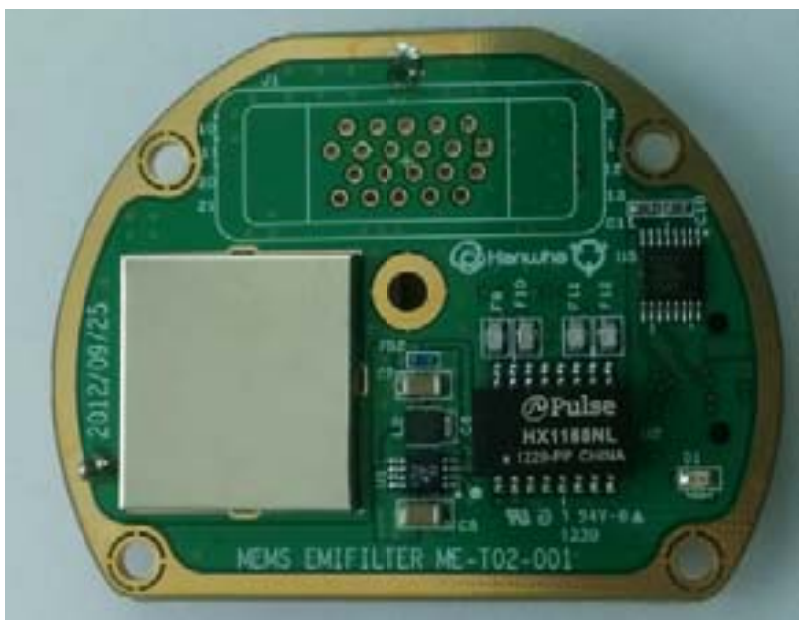

Fig. 6. Shape of the EMI filter board.

$\Phi 60 \mathrm{~mm}$. To prevent the cross talk between signals that could occur in the process of size minimization, it was designed based on a signal integrity analysis. A small highperformance navigation computer was developed by the optimal arrangement of parts and by the manufacture of high-integration/high-density build-up PCB. Fig. 5 shows the structure and shape of the navigation computer.

In the case of a navigation system, electromagnetic waves from the asymmetric noise component of power and external interface signals could cause abnormal operation of electronic boards within the navigation system or could affect adjacent devices. To prevent this, it was designed so that all signals could go through the EMI filter board. The EMI filter board was designed to satisfy the military standard (MIL-STD-461E). The filter was designed for rated capacity of the navigation system. A Y-type capacitor structure considering differential mode and an X-type capacitor considering common mode were designed and applied. The signal filter has a C-type low-pass filter structure considering the operating frequency. Also, a size minimization design was performed through the application of small parts, as shown in Fig. 6.

The housing of the MEMS-IMU/GPS integrated navigation system was designed so that it could tolerate high impact unlike general housing. To minimize the plastic deformation that could occur when high impact is applied, a material with high yield strength was used, and a mechanical rigidity reinforcement design for the weak parts that had been identified through a high impact analysis was applied. In addition, based on the high impact analysis of the internal electronic board components, a mechanical reinforcement design for the electronic boards having the possibility of damage was also reflected.

An MEMS inertial sensor has an inherent characteristic

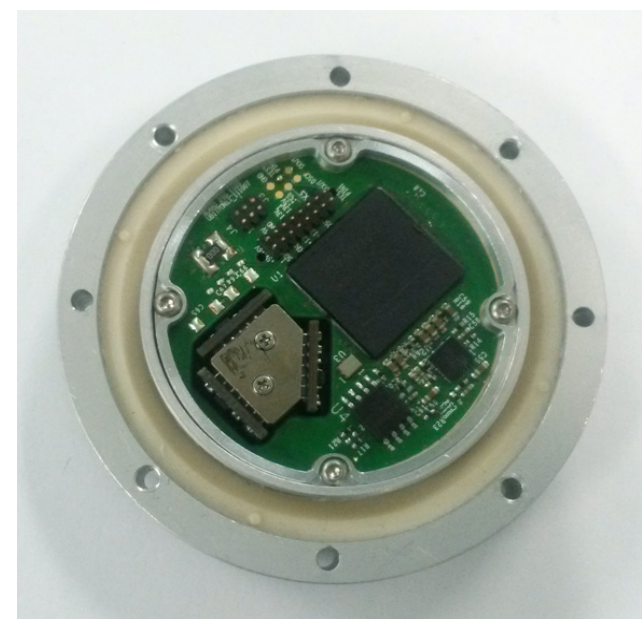

Fig. 7. Shape of the combining of the MEMS-ISA and the vibration isolator.

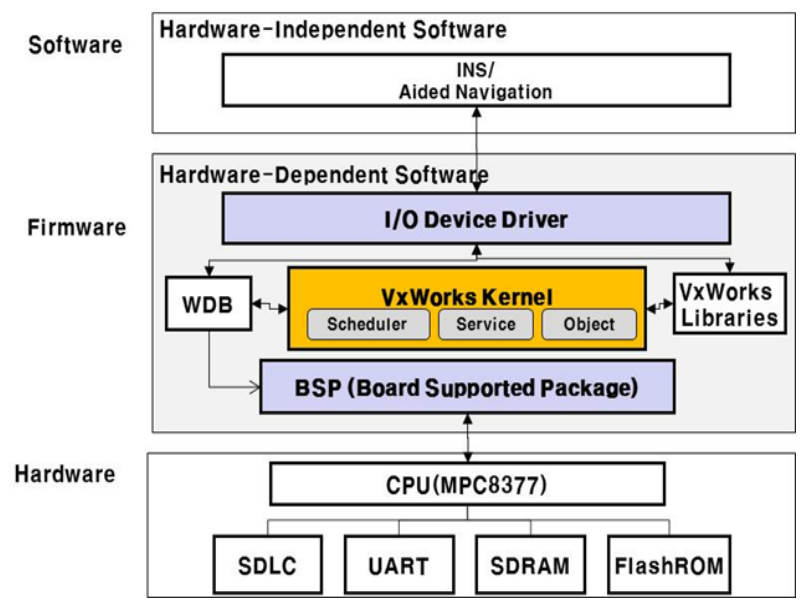

Fig. 8. Structure of the integrated navigation real-time SW platform.

where abnormal operation could occur when vibration is above a certain level. Thus, a vibration isolator should be applied to the navigation system. To satisfy the optimal vibration isolation frequency that had been selected through a frequency response function (FRF) experiment, the vibration isolator was designed using a material and a structure that are not much affected by the changes in temperature. Also, to supplement the high-impact resistance that could be weakened due to the use of a material with low rigidity, a damper was applied. The vibration isolator is combined wrapping the MEMS-ISA, as shown in Fig. 7, and protects the MEMS inertial sensor from external vibration.

The software of the MEMS-IMU/GPS integrated navigation system was designed based on VxWorks, which is a real-time operating system, and Fig. 8 shows the structure. The integrated navigation algorithm was designed using a deep coupling method, which has superior electromagnetic wave disturbance resisting performance compared to 


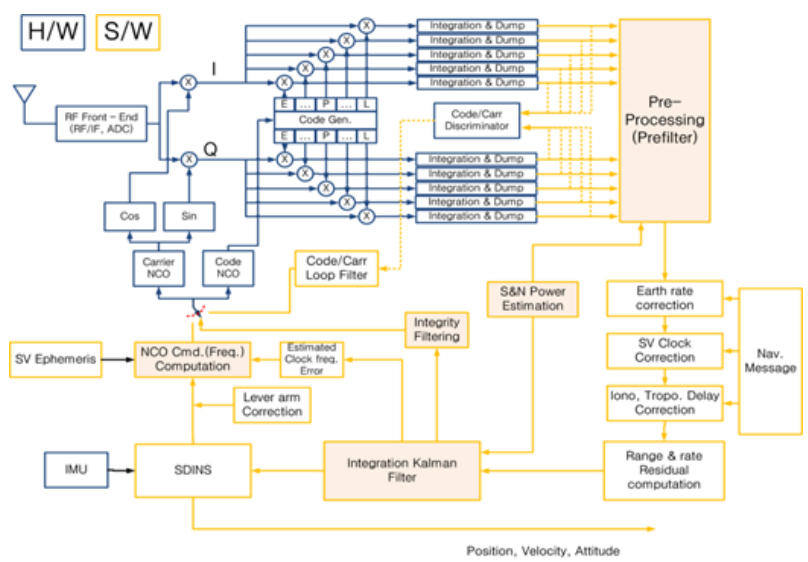

Fig. 9. Structure of the integrated navigation SW.

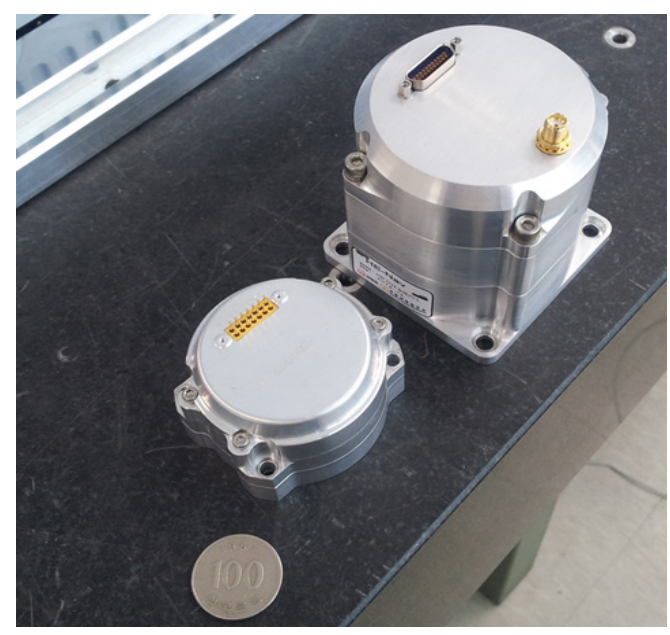

Fig. 10. Shape of the MEMS-IMU/GPS integrated navigation system.

existing weak coupling and strong coupling. Depending on the method of implementation, there are several kinds of deeply coupled integrated navigation algorithms. Horslund \& Hooker (1999) used a method in which range and rangerate residual are used for integrated navigation by estimating them from correlation values using code phase and carrier wave frequency discriminator. Beser et al. (2002) used a method in which pre-processing filtering is performed using a Kalman filter type discriminator. Gustafson et al. (2001) used a method in which a variable gain discriminator depending on $\mathrm{S} / \mathrm{N}$ is used as a pre-processing filter from ERC based correlator output. Besides, there are several kinds of known deep coupling algorithms, and they have different methods of pre-processing correlator output. In this study, an algorithm that uses ERC based correlator output and that improves the jamming signal mitigation performance by increasing integration time and using extra correlator output was designed. It was designed as a realtime multitasking structure so that GPS SW and integrated navigation SW could be operated in single CPU in real time. Fig. 9 shows the structure of the deeply coupled integrated navigation SW.

As described above, the MEMS-IMU/GPS integrated navigation system is a system where electronic, mechanical, software, and MEMS technologies are integrated. It was designed so that high performance, small size, environmental resistance, and reliability could be secured. M\&S verification for the design content was performed before the manufacture of a the system, and the MEMSIMU/GPS integrated navigation system was developed and manufactured through verification and debugging processes based on the manufacture of a prototype. Fig. 10 shows the shape of the developed MEMS-IMU/GPS integrated navigation system. The MEMS-ISA and signal processing board that correspond to the lower part of the navigation system can also be used as MEMS-IMU by separate housing, as shown on the left side of Fig. 10.

\section{PERFORMANCE OF THE MEMS-IMU/GPS INTEGRATED NAVIGATION SYSTEM}

\subsection{Inertial Sensor and Navigation Performance}

The performance of the MEMS-IMU/GPS integrated navigation system can be divided into inertial sensor performance and navigation performance. The inertial sensor performance is an important element that determines the performance of the navigation system when IMU is independently used or GPS is disconnected. Table 3 summarizes the measurements of the bias and noise of the MEMS gyroscope and accelerometer for a number of the system. The bias repeatability, which is the most important performance, was less than $20 \mathrm{deg} / \mathrm{hr}$ and $2 \mathrm{mg}$, respectively, which showed performance comparable to those of the foreign products.

The evaluation of the navigation performance was conducted through a vehicle test. Fig. 11 shows the vehicle test configuration and the position output trajectory of the integrated navigation system. The position error was

Table 3. MEMS inertial sensor performance results.

\begin{tabular}{cccc}
\hline Classification & Bias repeatability & Bias stability & Random Walk \\
\hline Gyroscope & $>20[\mathrm{deg} / \mathrm{hr}]$ & $>10[\mathrm{deg} / \mathrm{hr}]$ & $>0.2[\mathrm{deg} / \sqrt{\mathrm{hr}}]$ \\
Accelerometer & $>2[\mathrm{mg}]$ & $>1.5[\mathrm{mg}]$ & $>0.2[\mathrm{~m} / \mathrm{s} / \sqrt{\mathrm{hr}}]$ \\
\hline
\end{tabular}

Table 4. Vehicle test results (Position error (CEP).

\begin{tabular}{lllll}
\hline Test \# 1 & Test \#2 & Test \#3 & Test \#4 & Test \#5 \\
\hline $4.06 \mathrm{~m}$ & $7.57 \mathrm{~m}$ & $6.28 \mathrm{~m}$ & $6.71 \mathrm{~m}$ & $7.12 \mathrm{~m}$ \\
\hline
\end{tabular}




\section{Ref. GPS Antenna}

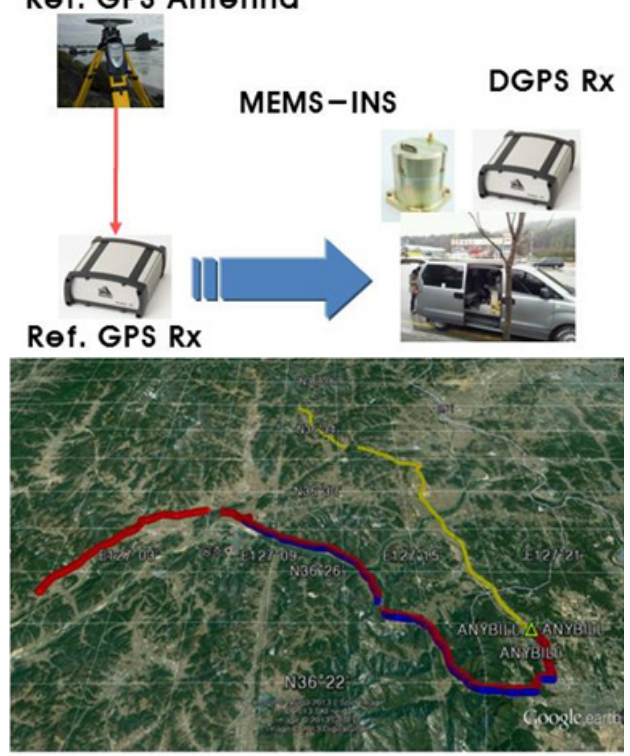

Fig. 11. Vehicle test configuration.

calculated in a dynamic condition based on precise DGPS. Table 4 summarizes the position error (CEP) for each test. It was shown that a position accuracy of about $10 \mathrm{~m}$ (CEP) could be obtained.

One of the important characteristics of the MEMSIMU/GPS integrated navigation system is the application of deeply coupled integrated navigation. This integrated navigation method has higher electromagnetic wave disturbance resisting performance (e.g., against jamming signals) than the existing integrated navigation method.
Fig. 12 shows the position error for each J/S compared to the existing coupling method. For the strong coupling method on the left side of the figure, the position error (CEP) abruptly increased when J/S was $29 \mathrm{~dB}$. However, for the deep coupling method on the right side, the position error was $10 \mathrm{~m}$ (CEP) level at the same J/S (29 dB), and a certain level of accuracy was maintained even at about $45 \mathrm{~dB}$.

\subsection{Environmental Resistance}

The reliability of the MEMS-IMU/GPS integrated navigation system can be examined by testing whether it normally operates in a number of harsh environments. The military standard defines this environmental resistance standard, and the resistance to various environments (e.g., high temperature/low temperature/vibration/impact/ humidity/altitude) is required. The MEMS-IMU/GPS integrated navigation system was designed so that it could tolerate harsh environmental conditions, and verification was performed through an environmental test. Figs. 13 and 14 show the results of the operation of the navigation system in high-temperature and low-temperature environments, respectively. The high-temperature test was performed at OO degrees above zero, and the low-temperature test was performed at $\mathrm{OO}$ degrees below zero. The navigation system operated normally at an accuracy of about $0.5 \sim 3.5 \mathrm{~m}$ even when it was exposed to the temperature environments for several hours. Fig. 15 shows the result of the operation in a vibration environment. The navigation system could be vulnerable to vibration due to the mechanical characteristic
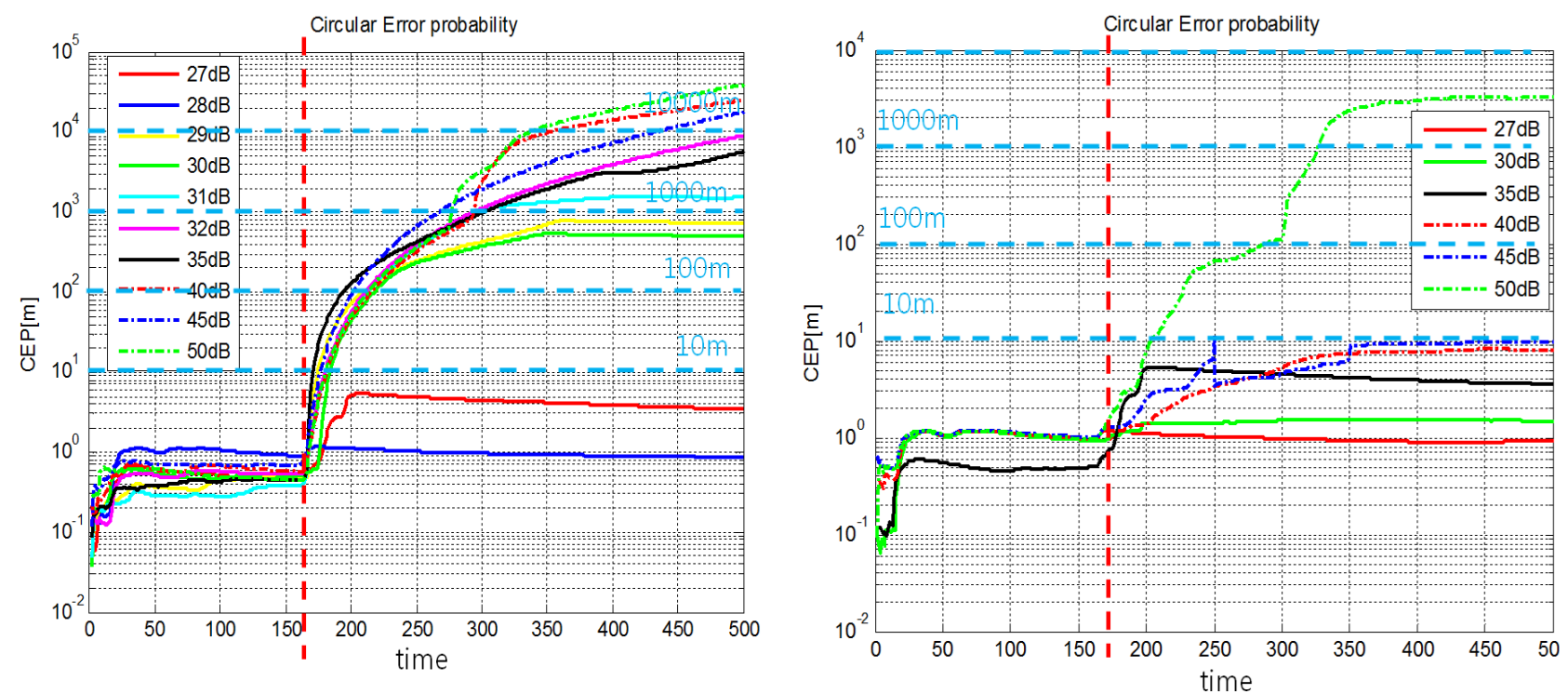

Fig. 12. Comparison of the position errors depending on the $\mathrm{J} / \mathrm{S}$. 


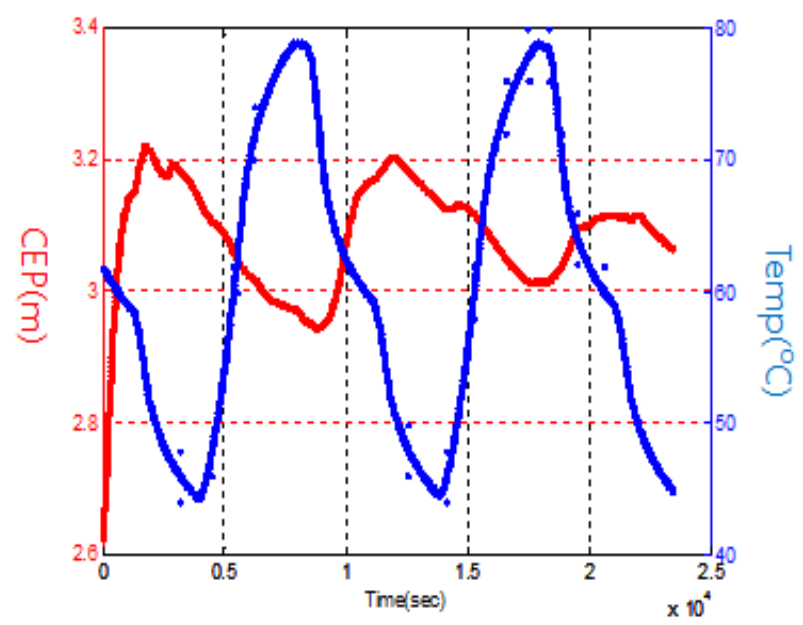

Fig. 13. Result of the high-temperature environmental test (Position error).

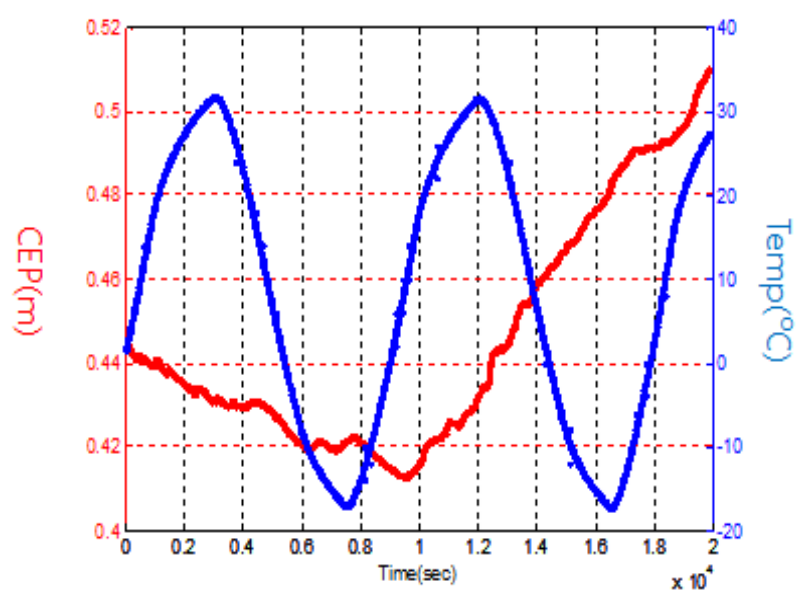

Fig. 14. Result of the low-temperature environmental test (Position error).

of the inertial sensor, unlike general electronic devices. The result indicated that the position error was less than $8 \mathrm{~m}$ (CEP) even when a vibration of more than $\mathrm{O}$ grms was applied at a frequency of $\mathrm{O} \sim \mathrm{O} \mathrm{kHz}$ for each axis. In

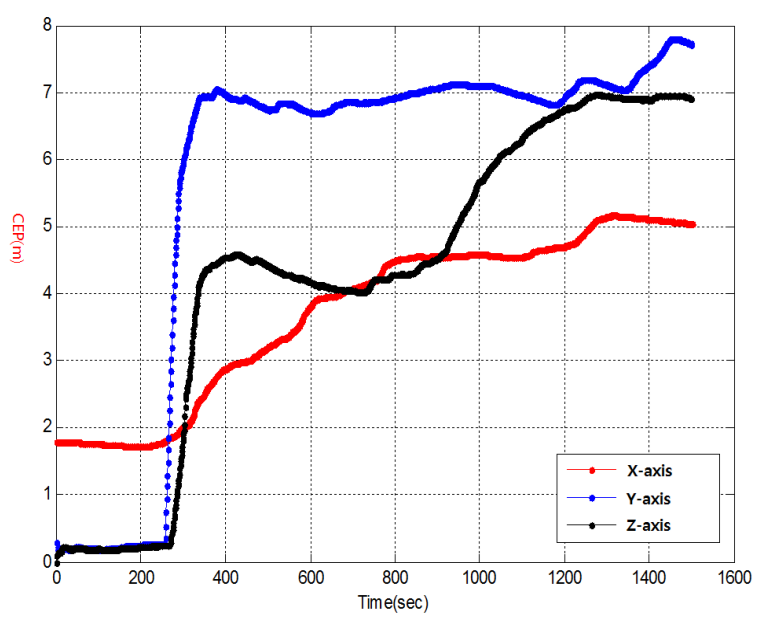

Fig. 15. Result of the vibration test (Position error).

addition, environmental resistance was verified through the environmental tests of shock, humidity, and altitude; and the resistance to EMI/EMC was also examined through a test.

\subsection{High-Shock Survivability}

One of the significant characteristics of the MEMS-IMU/ GPS integrated navigation system is high-shock survivability. This is a characteristic that only the navigation system using an MEMS sensor can possess; and the application to a smart projectile, which is one of the future weapon systems, is enabled only when it could tolerate the high shock during gun launching. All the components of the MEMS-IMU/GPS integrated navigation system were designed and developed for high- shock resistance. To examine the survivability, verification was performed using a gas-gun tester which can produce a high shock that is similar to that of gun launching. The system was loaded into the gas-gun shown in Fig. 16, and a high shock of more than OOOOO g was applied.

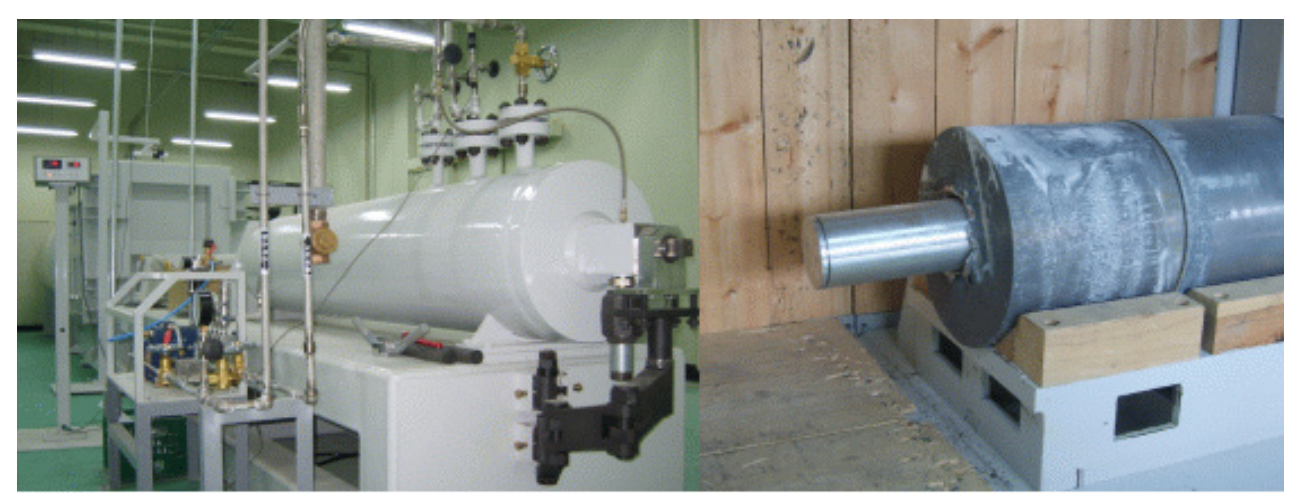

Fig. 16. High-impact gas-gun tester. 

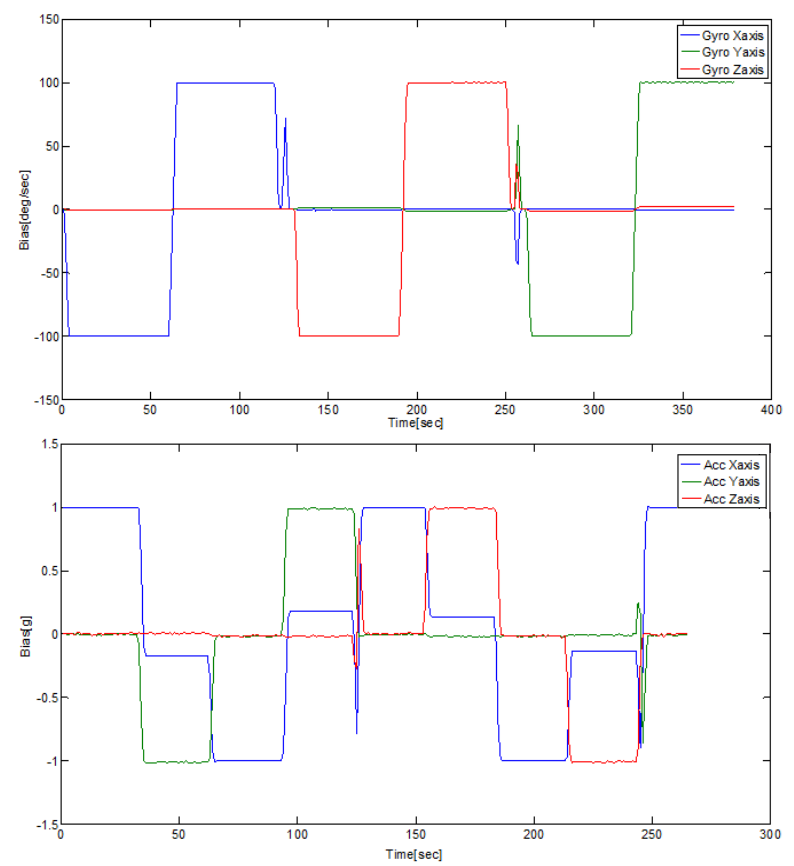

Fig. 17. MEMS sensor output after the high impact.

Then, whether it normally operates was examined. The result indicated that all the components (e.g., GPS receiver, navigation computer, etc.) were normal, and could perform integrated navigation. The MEMS sensor, which is relatively vulnerable compared to other components, also normally measured angular velocity and acceleration, as shown in Fig. 17.

\section{CONCLUSION}

In this paper, the design content and development result of an MEMS-based GPS/INS integrated navigation system were presented. For the suggested MEMS-IMU/ GPS integrated navigation system, a high-performance MEMS sensor was developed and applied, and the size was minimized through the ASIC of the major modules and the improvement in the integration density of the electronic boards. An optimal HW structure for deeply coupled integrated navigation was designed by adopting a single CPU structure. Based on this, the efficiency of HW resource usage was increased. Also, the design for environmental resistance and high-impact resistance, and its verification was performed through an environmental test and a gasgun test. This MEMS-IMU/GPS integrated navigation system satisfies all the performances and environmental resistance of a navigation system for weapon systems, and thus is expected to have high utility in various weapon systems.

\section{ACKNOWLEDGMENTS}

This research was conducted through the 'MEMS-based micro navigation system (contract number: UC100051FD)' project. The authors would like to thank the related persons in the Defense Acquisition Program Administration and the Agency for Defense Development and the participating researchers in Hanwha Corporation/R\&D Center, Navcours Co., Ltd., and Ubitronix Co., Ltd.

\section{REFERENCES}

Barbour, N. 2001, MEMS for Navigation, Proceedings of National Technical Meeting of Institute of Navigation (ION NTM 2001), pp.25-45

Beser, J., Alexander, S., Crane, R., \& Wyman, J. 2002, TRUNAV: Low-Cost Guidance/Navigation Unit Integrating a SAASM-Based GPS And MEMS IMU In a Deeply coupled Mechanization, Proceedings of Institute of Navigation GPS (ION GPS 2002), pp.545-555

Brown, T. G. 2003, Harsh Military Environments and Electromechanical (MEMS) Devices, Proceedings of IEEE Position Location and Navigation Symposium 2003, pp.753-760

Dowdle, J. R. \& Flueckiger, K. W. 1996, A GPS/INS Guidance System for Navy 5" Projectile, Proceedings of 52nd Annual Meeting of Institute of Navigation (ION AM 1996), pp.312-325

Gustafson, D. E., Dowdle, J. R., \& Elwell, Jr. J. M. 2001, Deeply Integrated Adaptive GPS-Based Navigator with Extended Range Code Tracking, US Patent 6,331,835 B1

Habibi, S., Cooper, S. J., Stauffer, J. M., \& Dutoit, B. 2008, Gun Hard Inertial Measurement Unit Based on MEMS Capacitive Accelerometer and Rate Sensor, Proceedings of IEEE Position Location and Navigation Symposium 2008, pp.232-237

Horslund, J. M. \& Hooker, J. R. 1999, Increase Jamming Immunity by Optimizing Processing Gain for GPS/INS Systems, US Patent 5,983,160 (MA: Raytheon Company, Lexington)

Schmidt, G. T. 1999, INS/GPS Technology Trends for Military Systems, The Draper Technology Digest, 3, 5-13

Warnasch, A. \& Killen, A. 2002, Low Cost, High G, Micro Electro-Mechanical System (MEMS), Inertial Measurements Unit (IMU) Program, Proceedings of IEEE Position Location and Navigation Symposium, Apr. 2002, pp.299-305. http://dx.doi.org/10.1109/ PLANS.2002.998922 


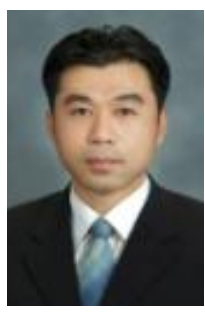

Jeong Won Kim received the Bachelor degree, Master degree and $\mathrm{Ph}$. D degree in Electronics engineering from Chugnam National University in 2002, 2004 and 2008. He is currently a chief research engineer at Defense R\&D Center, Hanwha Corp. His research interest includes GNSS receiver, GNSS/INS Integrated Navigation System.

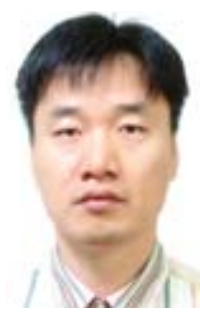

Chang Woo Nam received the Bachelor and Master degree in Electronic Engineering, Soongsil University in 1989, 1991. He is a doctoral course student at Electronic Engineering, Chungnam National University. He is currently a Chief Research Engineer at Defense R\&D Center, Hanwha Corp. His research interest includes GNSS receiver, Inertial Navigation System.

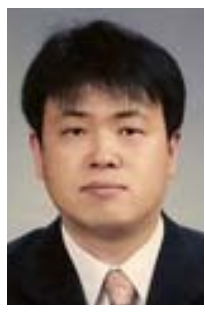

Jae-Cheul Lee received the $\mathrm{Ph}$. D degree in Institute of Optics from University of Rochester, USA in 1990. He has been with Hanwha Corp. since 2007 and also System Engineering Department, A-Jou University as an adjunct professor since 2000 . His research interests include inertial sensors, positioning \& navigation system and laser ranging system development.

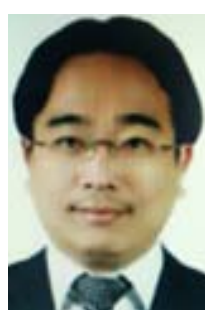

Sung Jin Yoon is a $\mathrm{Ph}$. D candidate of Electronics Engineering in A-Jou University. He is currently a senior researcher of Agency for Defense Development in Korea. His research interest includes inertial MEMS sensor and application of MEMS.

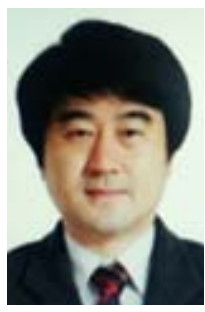

Jaewook Rhim received Ph. D degree in Aerospace Engineering from Graduate School of the University of Maryland, 1996. He is currently a principal researcher of Agency for Defense Development in Korea. His research interest includes inertial sensor and application of sensor. 